\title{
A Rare Presentation of Angina and Arrhythmia in Absent Left Main Coronary Artery
}

Shoaib Ashraf ${ }^{1}$, Syeda Hafsah Salman ${ }^{2}$, Nisha Ali ${ }^{2}$, Sarthak Kulshreshtha ${ }^{2}$, Muhammad Saad ${ }^{3}$

1. Internal Medicine, Bronx Care Health System Affiliated with Icahn School of Medicine at Mount Sinai, Bronx, USA 2. Internal Medicine, Bronx Care Health System, Bronx, USA 3. Cardiology, Bronx Care Health System Affiliated with Icahn School of Medicine at Mount Sinai, Bronx, USA

Corresponding author: Shoaib Ashraf, sashraf@bronxcare.org

\begin{abstract}
Coronary artery anomalies (CAAs) are congenital disorders with multiple variations in the number, shape, and location of the Ostia of the coronary arterial system. The congenitally absent left main coronary artery (LMCA) is a rare anomaly that can present with benign or fatal complications ahead in life. Diagnosis and management of CAAs are sometimes challenging in low-risk patients.
\end{abstract}

We present a unique case report of a 69-year-old Hispanic female who presented to the hospital with exercise-induced arrhythmia and angina symptoms. The patient complained of several episodes of chest pain, dizziness, and palpitations for a duration of two months. Electrocardiogram (EKG) and nuclear stress tests were equivocal. The angiogram revealed the separate origin of the left anterior descending artery (LAD) and left circumflex coronary artery (LCX) from the left coronary sinus. This anomaly should be considered in differentials when evaluating patients with angina symptoms.

Congenital absence of LMCA is a rare condition that remains asymptomatic in the majority of the cases. It can present with exertional chest pain, palpitations, syncope, and sudden cardiac death (SCD). Occurrences of angina and arrhythmia should be carefully evaluated, and symptoms should be followed up closely. A coronary angiogram and electrophysiological testing can assist in the diagnosis.

Review began 10/17/2020 Review ended 12/11/2020 Published 12/18/2020

๑) Copyright 2020

Ashraf et al. This is an open access article distributed under the terms of the Creative Commons Attribution License CC-BY 4.0., which permits unrestricted use, distribution, and reproduction in any medium, provided the original author and source are credited.
Categories: Cardiology, Internal Medicine, Anatomy

Keywords: congenital absent left main coronary artery, angina, cardiac arrhythmia, coronary angiography

\section{Introduction}

Coronary artery anomalies (CAAs) are congenital disorders characterized by multiple variations in the number, shape, and location of the Ostia of the coronary arteries. CAAs can be benign or life-threatening. It can present with a wide variety of symptoms such as chest pain, myocardial infarction, arrhythmias, sudden death, syncope, and congestive heart failure. Early detection of such an anomaly is of great significance as it can help to prevent serious complications. This article aims to highlight the rare causes of angina and arrhythmia. Our patient was an unusual case of congenital absence of the left main coronary artery (LMCA) who presented with angina symptoms (Video 1).

\section{Case Presentation}

A 69-year-old Hispanic woman with a past medical history of hypertension, dyslipidemia, and celiac disease was admitted to the hospital from the cardiology clinic after experiencing exercise-induced angina symptoms. Her primary care physician had referred her to the cardiology clinic on account of two months history of several episodes of chest pain, dizziness, and palpitations. Chest pain was intermittent, nonradiating, and was associated with exertion at times. She denied syncope, shortness of breath, nocturnal dyspnea, and heartburn. Her medications included aspirin, atorvastatin, carvedilol, losartan, and omeprazole. She had a family history of leukemia, coronary artery disease (CAD), hypertension, and dyslipidemia. She denied smoking, alcohol, and the use of illicit drugs. She was scheduled for a pharmacological nuclear stress test to evaluate for CAD. During the pharmacological nuclear stress test, she developed angina and palpitations. The nuclear stress test was equivocal. Vital signs showed a heart rate of 160 beats per minute (bpm), oxygen saturation of $98 \%$ on ambient air, and blood pressure of 136/82 $\mathrm{mmHg}$. The patient received $200 \mathrm{mg}$ IV aminophylline for a possible regadenoson adverse reaction. On physical examination, she was ill-appearing and was in moderate distress. The respiratory and neurologic exams were unremarkable. Her 12-lead electrocardiography (EKG) showed sinus arrhythmia with a heart rate of 99 bpm. The PR interval and QTc measured $142 \mathrm{~ms}$ and $441 \mathrm{~ms}$, respectively (Figure 1). 


\section{Cureus}

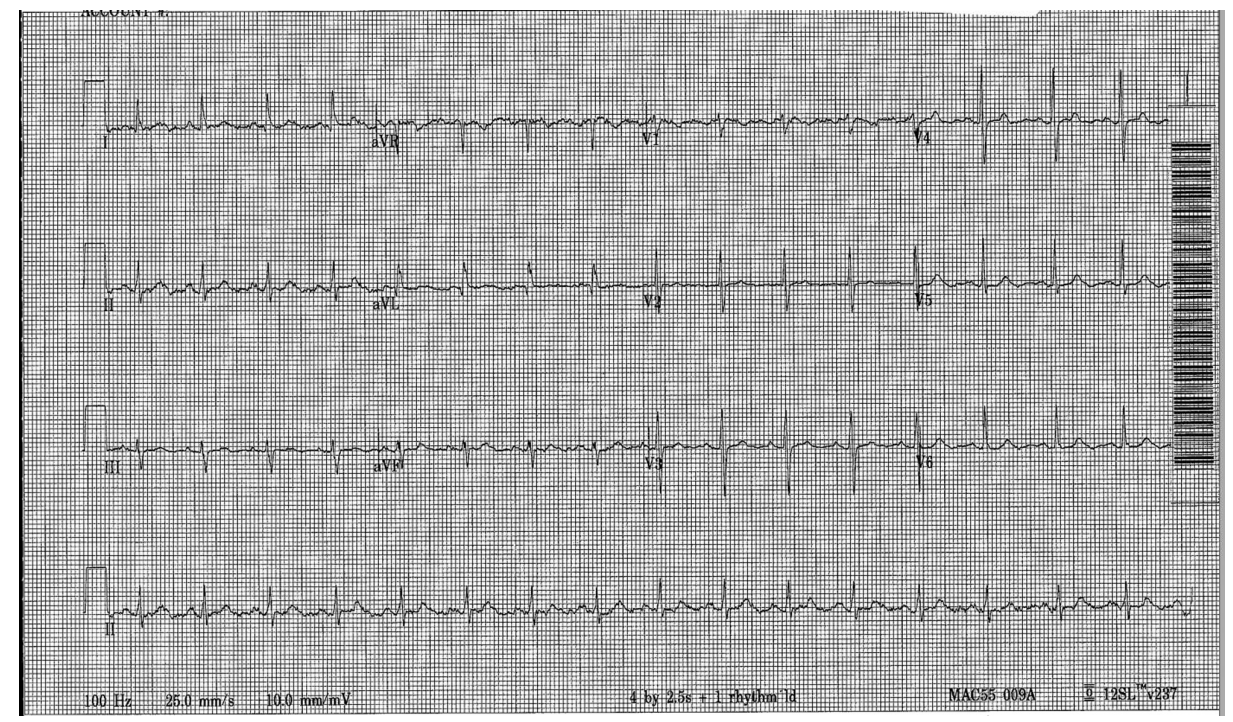

FIGURE 1: Electrocardiography of the case

The 12-lead electrocardiography showed a normal sinus rhythm with a heart rate of 99 beats per minute

Laboratory investigations initially showed elevated high-sensitivity troponins of $33 \mathrm{ng} / \mathrm{ml}$ and a repeat value of $29 \mathrm{ng} / \mathrm{ml}$ with a delta of $-4 \mathrm{ng} / \mathrm{ml}$ on serial measurements (normal limit: $<12 \mathrm{ng} / \mathrm{ml}$ ). The patient had normal myocardial perfusion images on the nuclear stress test (Figure 2), but because of equivocal EKG changes, the patient underwent cardiac catheterization. The coronary angiogram revealed no significant atherosclerotic lesion or fixed stenosis (Video 1, Video 2), but the LMCA was absent.

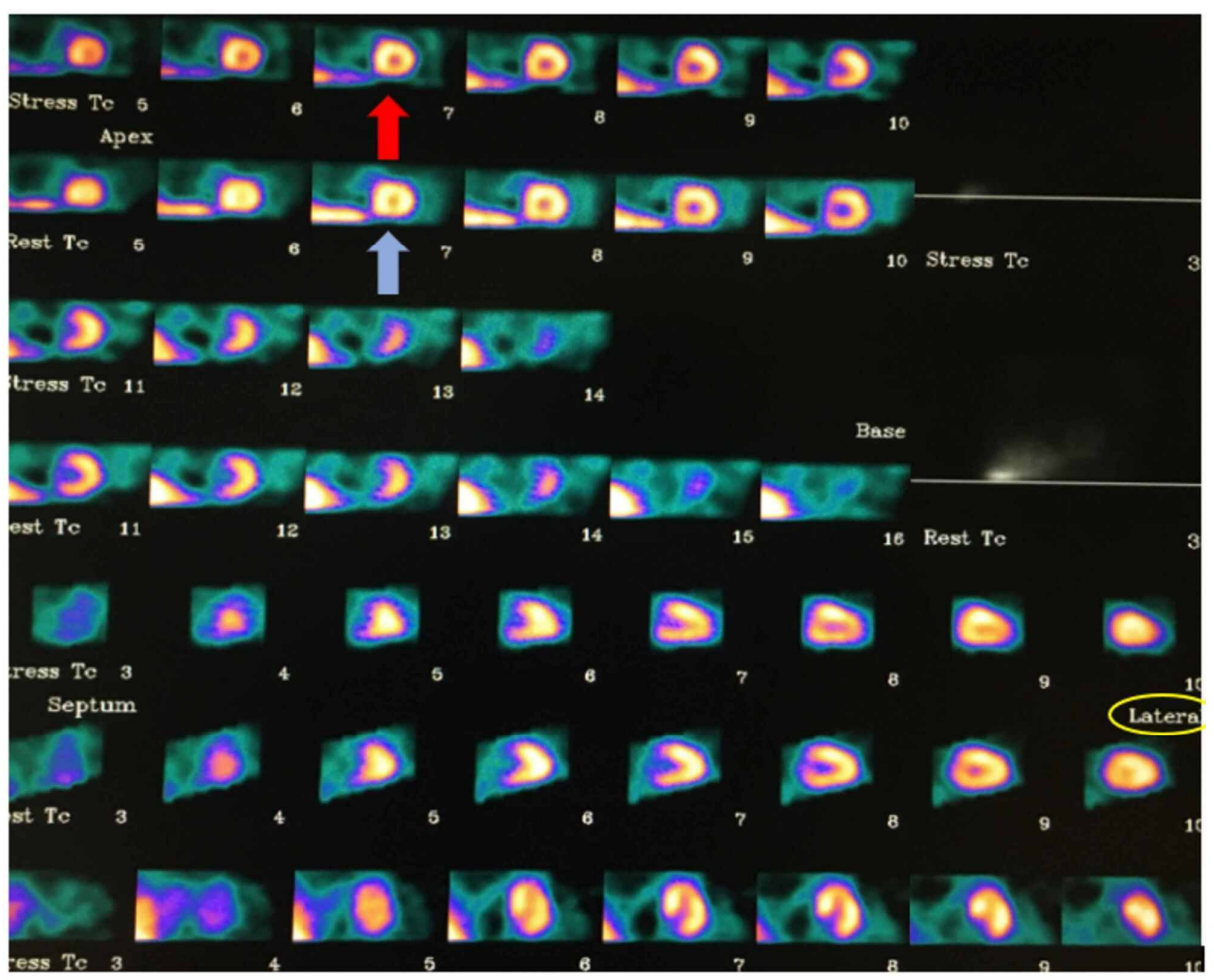

\section{FIGURE 2: Nuclear stress test images of the case}

The nuclear stress test showed normal myocardial perfusion in short axis, vertical long-axis, and horizontal long-axis views in both rest and stress images. The red arrow indicates normal perfusion in the stress phase. The blue arrow indicates normal perfusion in the rest phase. The yellow circle shows the lateral view of the nuclear stress test 


\section{Cureus}

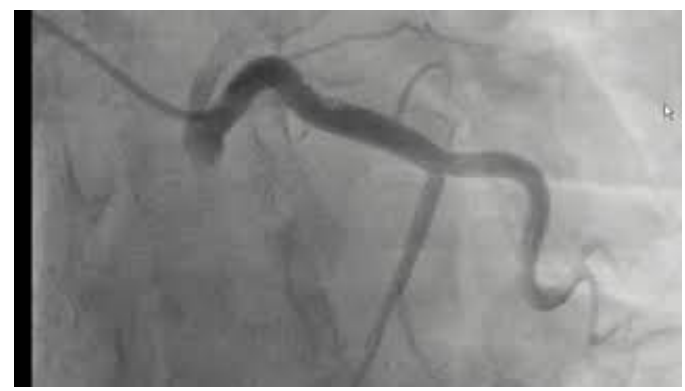

\section{VIDEO 1: Left heart catheterization - view 1}

Left anterior oblique (LAO) with caudal views showing absent left main coronary artery. The left circumflex artery shown here is patent. It is arising from the aorta directly

View video here: https://youtu.be/GS7n_-NztdE

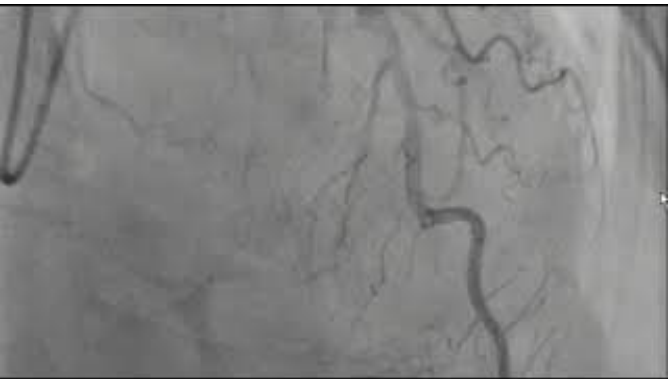

\section{VIDEO 2: Left heart catheterization - view 2}

Right anterior oblique (RAO) cranial view of left heart catheterization showing absent left main and patent LAD and LCX

LAD: left anterior descending artery; LCX: left circumflex artery

View video here: https://youtu.be/wm6Jko1tIrE

The angiogram revealed the separate origin of the left anterior descending artery (LAD) and left circumflex coronary artery (LCX) from the left coronary sinus (Video 1, Video 2). The patient was diagnosed with a congenital absence of LMCA. Transthoracic echocardiogram prior to admission revealed a left ventricular ejection fraction (LVEF) of 73.5\%, estimated right ventricular systolic pressure of $40.69 \mathrm{mmHg}$, and grade 1 diastolic dysfunction (Video 3, Video 4). The patient did not undergo a coronary flow reserve test by echocardiography due to low suspicion of coronary anomalies. The patient had undergone Holter monitor EKG testing a few years prior to this presentation due to a similar episode of palpitations and chest pain, but the results were not available.

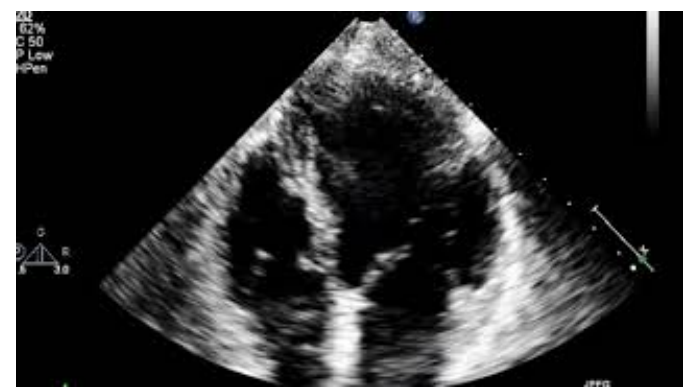

\section{VIDEO 3: Transthoracic echocardiogram - view 1}

Apical four-chamber view of transthoracic echocardiogram showing normal wall motion and contractility

View video here: https://youtu.be/uKAg7yeh-YY 


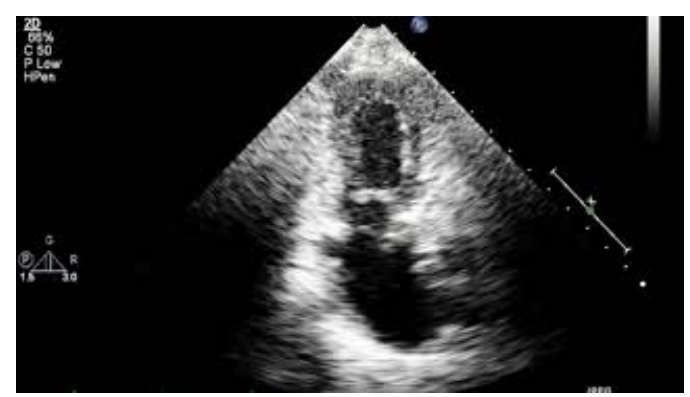

\section{VIDEO 4: Transthoracic echocardiogram - view 2}

Apical two-chamber view of transthoracic echocardiogram showing normal anterior and inferior wall contractility

View video here: https://youtu.be/JpFuGoCUPkE

Medical management was primarily considered with conventional anti-angina and anti-hypertensive medications with risk factor modification. An event monitor was provided to rule out any abnormal electrical activity or electrical pathways that can lead to sudden cardiac death (SCD). The patient was discharged two days after admission.

\section{Discussion}

The natural anatomy of coronary arteries commences from the aorta and its course to converge towards the cardiac apex. Generally, it consists of two primary branches, which include the right coronary artery (RCA) and the left coronary artery (LCA). The LCA immediately bifurcates into LAD and LCX, which supply the major areas of the left ventricle and the interventricular septum [1-3]. CAA is the aberrant origination of the coronary artery and/or its branches as opposed to the natural course. It is a rarely reported condition, observed in $0.6 \%-1.3 \%$ of patients in routine angiographic series and $1 \%$ of routine autopsies [3]. Coronary anomalies may include anomalies in its origin (coronary aneurysm, absent coronary artery), intramural coronary artery, subendocardial coronary course, split RCA, hypoplastic coronary artery, anomalies of coronary termination, and anastomotic vessels. The absence of LMCA is the most common CAA, with an incidence of approximately $0.5 \%$ [2]. In absent LMCA, LAD and LCX originate separately through the aortic sinus. Another commonly found anatomic variant is the origin of LCX from RCA instead of LMCA, with a prevalence of $0.37 \%$ [2,4]. It has a predilection for males than females [2]. Embryonically, anomalies in the ectopic growth of the coronary sinus due to defects in the signaling pathway are responsible for the absence of LMCA. It is considered a benign condition with the normal distribution of vessels and no clinical or hemodynamic compromise. The risk of SCD is considered rare in an incidentally discovered coronary anomaly, but it still remains unknown. The risk of SCD is considerably higher when LCX or LAD passes through the aorta and pulmonary artery. However, anomalies of great vessels of the heart have been observed when LCMA originates through the posterior sinus of Valsalva [5,6].

In an asymptomatic or quiescent state, it is usually detected incidentally by CT or invasive coronary angiography $[7,8]$. Although echocardiography has been proposed as a modality to detect coronary anomalies in severe cases, it is not utilized as a diagnostic modality due to very limited evaluation [8]. It is imperative to estimate and outline the coronary artery origin, distribution, and course whenever CAA is suspected. The standard practice is the use of coronary angiogram, but it is considered limited owing to its two-dimensional imaging and invasive nature $[9,10]$. Although coronary CT is considered a diagnostic modality to visualize coronary anatomy, the use of radiation and contrast makes its use limited, especially in women of childbearing age. CMR can be utilized safely in this population, but due to its limitation in evaluating small coronary branches, a substantial number of cases can be missed [11,12].

In our case, the patient developed angina symptoms in the post-stress period as a part of stress testing along with the run of non-sustained ventricular tachycardia. Her stress test was equivocal. This event may raise a suspicion for occlusive $\mathrm{CAD}$, anatomic variation in coronary arteries, structural variation such as myocardial bridging, and microvascular angina. The coronary angiography revealed patent coronaries with absent LMCA and the origin of LAD and LCX from the left coronary sinus. Our case is unique because the patient presented with angina and tachyarrhythmia and was found to have an absent LMCA. In contrast, an absent LMCA is reported to have a benign clinical course, and most patients remain asymptomatic. Although we excluded myocardial bridging and acute coronary syndrome (ACS), microvascular ischemia could not be completely ruled out. Published data shows that there might be an association between an anomalous coronary artery and an embryonic defect at the cellular level leading to microvascular ischemia [13]. Upon review of the literature, it was found that absent LMCA can present with angina symptoms, but its presentation with syncope and arrhythmia is a rare phenomenon [14-17]. There are no standardized guidelines regarding the management of this condition [18]. However, caution should be taken during coronary angiography to avoid the misdiagnosis of coronary artery atresia in the presence of separate Ostia 


\section{Conclusions}

Congenital absence of LMCA is a rare condition that remains asymptomatic in the majority of the cases. It can present with exertional chest pain, palpitations, syncope, and SCD. Occurrences of angina and arrhythmia should be carefully evaluated, and symptoms should be followed up closely. A coronary angiogram and electrophysiological testing can assist in the diagnosis of coronary anomalies.

\section{Additional Information \\ Disclosures}

Human subjects: Consent was obtained by all participants in this study. Conflicts of interest: In compliance with the ICMJE uniform disclosure form, all authors declare the following: Payment/services info: All authors have declared that no financial support was received from any organization for the submitted work. Financial relationships: All authors have declared that they have no financial relationships at present or within the previous three years with any organizations that might have an interest in the submitted work. Other relationships: All authors have declared that there are no other relationships or activities that could appear to have influenced the submitted work.

\section{References}

1. Kardos A, Babai L, Rudas L, et al.: Epidemiology of congenital coronary artery anomalies: a coronary arteriography study on a central European population. Cathet Cardiovasc Diagn. 1997, 42:270-275. 10.1002/(sici)1097-0304(199711)42:3<270::aid-ccd8>3.0.c0;2-9

2. Kastellanos S, Aznaouridis K, Vlachopoulos C, Tsiamis E, Oikonomou E, Tousoulis D: Overview of coronary artery variants, aberrations and anomalies. World J Cardiol. 2018, 10:127-140. 10.4330/wjc.v10.i10.127

3. Yilmaz-Cankaya B, Kantarci M, Yalcin A, Durur-Karakaya A, Yuce I: Absence of the left main coronary artery: MDCT coronary angiographic imaging. Eurasian J Med. 2009, 41:56-58.

4. Cheezum MK, Liberthson RR, Shah NR, Villines TC, O'Gara PT, Landzberg MJ, Blankstein R: Anomalous aortic origin of a coronary artery from the inappropriate sinus of Valsalva. J Am Coll Cardiol. 2017, 69:15921608. 10.1016/j.jacc.2017.01.031

5. Pereira da Costa Sobrinho O, Dantas de Lucena J, Silva Pessoa R, et al.: Anatomical study of length and branching pattern of main trunk of the left coronary artery. Morphologie. 2019, 103:17-23. 10.1016/j.morpho.2018.10.002

6. Young PM, Gerber TC, Williamson EE, Julsrud PR, Herfkens RJ: Cardiac imaging: part 2, normal, variant, and anomalous configurations of the coronary vasculature. AJR Am J Roentgenol. 2011, 197:816-826. 10.2214/AJR.10.7249

7. Abedin Z, Goldberg J: Origin and length of left main coronary artery: its relation to height, weight, sex, age, pattern of coronary distribution, and presence or absence of coronary artery disease. Cathet Cardiovasc Diagn. 1978, 4:335-340. 10.1002/ccd.1810040318

8. Yildiz A, Okcun B, Peker T, Arslan C, Olcay A, Bulent Vatan M: Prevalence of coronary artery anomalies in 12,457 adult patients who underwent coronary angiography. Clin Cardiol. 2010, 33:E60-E64. 10.1002/clc. 20588

9. Ajayi NO, Lazarus L, Vanker EA, Satyapal KS: Absent left main coronary artery with variation in the origin of its branches in a South African population. Anat Histol Embryol. 2015, 44:81-85. 10.1111/ahe.12109

10. Yamanaka O, Hobbs RE: Coronary artery anomalies in 126,595 patients undergoing coronary arteriography . Cathet Cardiovasc Diagn. 1990, 21:28-40. 10.1002/ccd.1810210110

11. Frommelt PC, Frommelt MA, Tweddell JS, Jaquiss RD: Prospective echocardiographic diagnosis and surgical repair of anomalous origin of a coronary artery from the opposite sinus with an interarterial course. J Am Coll Cardiol. 2003, 42:148-154. 10.1016/s0735-1097(03)00503-5

12. Maron BJ, Carney KP, Lever HM, Lewis JF, Barac I, Casey SA, Sherrid MV: Relationship of race to sudden cardiac death in competitive athletes with hypertrophic cardiomyopathy. J Am Coll Cardiol. 2003, 41:974980. 10.1016/s0735-1097(02)02976-5

13. Pérez-Pomares JM, de la Pompa JL, Franco D, et al.: Congenital coronary artery anomalies: a bridge from embryology to anatomy and pathophysiology--a position statement of the development, anatomy, and pathology ESC Working Group. Cardiovasc Res. 2016, 109:204-216. 10.1093/cvr/cvv251

14. Chaosuwannakit N: Anatomical variants and coronary anomalies detected by dual-source coronary computed tomography angiography in North-eastern Thailand. Pol J Radiol. 2018, 83:e372-e378. 10.5114/pjr.2018.78420

15. Lorber R, Srivastava S, Wilder TJ, et al.: Anomalous aortic origin of coronary arteries in the young: echocardiographic evaluation with surgical correlation. JACC Cardiovasc Imaging. 2015, 8:1239-1249. 10.1016/j.jcmg.2015.04.027

16. Schroeder S, Achenbach S, Bengel F, et al.: Cardiac computed tomography: indications, applications, limitations, and training requirements: report of a Writing Group deployed by the Working Group Nuclear Cardiology and Cardiac CT of the European Society of Cardiology and the European Council of Nuclear Cardiology. Eur Heart J. 2008, 29:531-556. 10.1093/eurheartj/ehm544

17. Warnes CA, Williams RG, Bashore TM, et al.: ACC/AHA 2008 guidelines for the management of adults with congenital heart disease: a report of the American College of Cardiology/American Heart Association Task Force on Practice Guidelines (Writing Committee to Develop Guidelines on the Management of Adults With Congenital Heart Disease). Developed in Collaboration With the American Society of Echocardiography, Heart Rhythm Society, International Society for Adult Congenital Heart Disease, Society for Cardiovascular 


\section{Cureus}

Angiography and Interventions, and Society of Thoracic Surgeons. J Am Coll Cardiol. 2008, 52:e143-e263. 10.1016/j.jacc.2008.10.001

18. Van Hare GF, Ackerman MJ, Evangelista JK, et al.: Eligibility and disqualification recommendations for competitive athletes with cardiovascular abnormalities: Task Force 4: Congenital Heart Disease: A Scientific Statement From the American Heart Association and American College of Cardiology. J Am Coll Cardiol. 2015, 66:2372-2384. 10.1016/j.jacc.2015.09.036 